\title{
Effect of Knowledge Retention From the First Principles Course on Performance in the Second Principles Course
}

\author{
Rebecca Hutchins \\ Appalachian State University \\ Gerald L. Hutchins \\ Appalachian State University
}

We explore the impact retention of specific concepts from the first course in accounting principles has on student performance in the subsequent course. There has been worthwhile research on the effect of general factors such as prior performance and knowledge. We extend this work by considering how gaps in students' understanding alter their success in a subsequent course. In these analyses we find a student's inability to demonstrate understanding of key concepts from the financial accounting course are significantly related to important learning objectives in the second course. These findings suggest potential areas to re-enforce early in the managerial course.

Keywords: knowledge retention, performance, principles, assets

\section{INTRODUCTION}

The importance of Accounting Principles as a core component of the education of business majors is widely accepted. Most universities require business students take two accounting Principles classes, most commonly with Financial Accounting the primary content of the first class and Managerial Accounting the second. (Doran, et.al., 1991) The success of the first course in preparation of students for future success in accounting has been the focus of many groups within the accounting profession, including the Accounting Education Change Commission (AECC, 1992), the American Accounting Association (AAA, 1986) and the major accounting firms (Arthur Andersen \& Co. et al., 1989). Individual accounting educators have also discussed the role of the introductory course in the accounting and business curriculum (Baldwin \& Ingram, 1991; Pincus, 1997; Vangermeersch, 1997). Continuing research has connected general academic factors such as prior academic success, often measured using GPA, prior knowledge of accounting (Papageorgiou \& Carpenter, 2019; Bryne \& Flood, 2008; Pasewark, 2020; Danko,et.al., 1992), and gender on success in accounting courses. ( Papageorgiou \& Halabi, 2014; Tan \& Laswad, 2008) The importance of the first course lies in its ability to both present useful accounting information that can lead to better decision-making for all business majors, and attract, or discourage, individuals from becoming accounting majors (Kaenzig $\&$ Keller, 2011). In this paper we look at the performance in the second course and how it relates to content retention from the first course. The study was conducted over three semesters, including a total of 231 students. The course is the second in the Principles of Accounting sequence, required of all majors in the College of Business. The class is primarily a Managerial accounting class, with the focus on determination 
of costs and prediction of costs for budgeting purposes. A review is conducted at the beginning of the class, covering Financial Statements (order of preparation; what each statement includes and how it is used); Account types (asset, liability, equity, revenue, and expense: recognize accounts in each category and on which financial statement each is reported); and the use of debits and credits (which accounts have a debit or credit balance).

\section{PRIOR LITERATURE}

Prior literature includes a significant number of studies that have been conducted to address various aspects of the introductory accounting course (Jordan, \& Samuels, 2020; Baldwin \& Ingram, 1991) proposed that the content and objectives of the Principles sequence be considered a priority. They ask the question "Have you ever heard anyone describe the objective of the elementary accounting sequence?" They propose that the "elementary accounting courses should be thought of as general education courses, primarily for the business major perhaps, but also of value to a wide variety of students across the campus. We suggest a complete revamping of content and pedagogy to serve the needs of the $80-85 \%$ of enrollees that will not become accounting majors, rather than the needs of the $15-20 \%$ of students who will."

The literature is full of proposals for how to reform the accounting curriculum (Cherry \& Mintz, 1996; Cherry \& Reckers, 1983). "For almost 30 years, there have been regular calls for accounting education change to meet the needs of the evolving profession." (Pincus et al, 2017; Warren \& Young, 2012) The focus of these authors addresses the tension between using the introductory accounting courses as a general business education class versus the pressure to recruit into the accounting major from these same classes. Also, many faculty still see these courses as preparatory for Intermediate Accounting, rather than seeing them as being most useful when the focus is on concepts rather than on procedures (calculation and recording of specific accounting items). To these authors teaching how to depreciate is less important than understanding the concepts involved in depreciation. Such topics as bank reconciliation are viewed as not reaching the objective of helping all students understand the larger picture of why reconciliation is important. Many studies have examined the determinants of student performance in the first accounting course and the effect on recruitment into accounting as a major (Kaenzig \& Keller, 2011; Geiger \& Ogilby, 2000).

The literature is rich in studies on the effect of prior content knowledge and prior academic success on student performance. Tan and Lasward (2008) found that prior content knowledge is an important predictor of success. In the context of this study, knowledge from the first principles course would be expected to be a factor in success in the second course. Byrne and Flood (2008) also found a positive effect on performance in the first accounting course in University when students had prior accounting content knowledge from pre-University academic courses.

The literature on student performance in online principles courses includes the research of Papageorgiou and Halabi (2014). Looking at determinants of student success in online accounting courses, these authors find academic aptitude and prior content knowledge as strong factors in success. The study included eight courses in accounting over the course of earning a degree, and found that as students progressed, the effect of pre-University content knowledge was reduced. The authors did not directly consider the effect of content in one University Accounting class on success in another University Accounting class.

\section{METHODOLOGY}

\section{Data Sources}

This paper uses course performance data from 231 students taking a managerial accounting course taught by one professor during spring, summer, or fall terms of 2020 at a medium size university in the southeastern United States. The University IRB office treated this as exempt from review because there was minimal student risk and the main intent was to improve instruction. The data include student performance on a Review Quiz, Exam One, the final, and overall course grade. Nearly all these students 
received only on-line instruction. One section of 28 students in the Spring term of 2020 received in-person instruction the first 10 weeks of the term. This section was transitioned to on-line instruction using on-line lectures and resources in mid-March due to university COVID safeguards. These students were not significantly different on key independent variables from those who received only on-line instruction. The exams were all multiple choice and done online with randomized question presentation. Both overall performance and performance on specific quiz and exam questions are analyzed.

\section{ANALYSIS}

\section{Weaknesses Identified}

To help students self-identify possible weaknesses in the knowledge and understandings of important concepts they had retained from basic accounting principles, they were required to complete a Review Quiz of thirty-four questions. After they received their quiz results, online resources were provided to help them better understand the items on which they had difficulty and to overcome deficits in their retention. They were able to retake the quiz a second time to confirm their mastery of the material. Students taking the quiz only once had higher scores (median $91.2 \%$ correct) than those who retook the quiz. The median correct score for the $56.3 \%$ of students who retook the quiz rose from $73.5 \%$ on the first try to $97.1 \%$ on the second try. This suggests students did benefit from reviewing their errors and used the online resources. A student's highest grade was worth up to 10 points out of 1,000 toward their final course grade.

TABLE 1

REVIEW QUIZ QUESTIONS CORRECT VS. INCORRECT

\begin{tabular}{|l|c|c|c|c|}
\hline & $\begin{array}{c}\mathbf{N} \\
\text { Correct }\end{array}$ & $\begin{array}{c}\mathbf{N} \\
\text { Incorrect }\end{array}$ & $\begin{array}{c}\text { Correct } \\
\%\end{array}$ & $\begin{array}{c}\text { Incorrect } \\
\%\end{array}$ \\
\hline R16 Select a purpose for which a journal is not useful & 106 & 108 & $49.5 \%$ & $50.5 \%$ \\
\hline R3 Calculate stockholder equity & 109 & 105 & $50.9 \%$ & $49.1 \%$ \\
\hline R19 Select the effect a paid dividend has on accounts & 122 & 91 & $57.3 \%$ & $42.7 \%$ \\
\hline R12 Identify asset accounts from list and total & 145 & 70 & $67.4 \%$ & $32.6 \%$ \\
\hline R33 Select an account increased by a debit & 149 & 67 & $69.0 \%$ & $31.0 \%$ \\
\hline R11 Identify asset accounts from list and total & 151 & 63 & $70.6 \%$ & $29.4 \%$ \\
\hline R8 Identify asset accounts from list and total & 152 & 61 & $71.4 \%$ & $28.6 \%$ \\
\hline R34 Identify credit accounts and total the amount & 154 & 61 & $71.6 \%$ & $28.4 \%$ \\
\hline R13 Select definition of chart of accounts & 157 & 58 & $73.0 \%$ & $27.0 \%$ \\
\hline R5 Identify asset accounts from list and total & 159 & 55 & $74.3 \%$ & $25.7 \%$ \\
\hline
\end{tabular}

Table 1 above highlights the ten questions on which students had the most difficulty on their first try at the Review Quiz. The error rates ranged from $25.7 \%$ up to $50.5 \%$ on these items. The questions are listed in the table with only the question number and general topic. Six of the ten questions required some calculations. Because "asset" is one of the central concepts within accounting, four of the questions asked the student to consider a list of accounts, identify asset accounts from the list and total them. Although 
mathematical ability (or fear) has been linked to success in accounting courses, it seems reasonable that simply adding a series of numbers from the list of accounts would not be affected by any inability to work math formulas. The other two calculation questions in the 'top ten' list involved the concepts of stockholder's equity and total credits. The remaining four questions asked students to demonstrate their understanding of basic accounting concepts: a chart of accounts, a journal, the effect of a paid dividend on accounts, and debits.

\section{Impact on Overall Course Performance}

Are early accounting courses entirely self-contained or do they build on one another? Table 2 and Table 3 show T-Tests results for the ten Review Quiz measures with the highest rates of incorrect responses on the percentage correct on the final exam and on overall course grades. (Note that all T-Tests were done as independent samples t-tests.) Our data suggest that the inability to correctly identify asset and credit accounts (R5 and R34) during the review phase of the managerial course is related to a lower percentage of correct responses on the final exam but not to lower overall course grades. The Review Quiz is given during the first two weeks of Principles Two course which suggests an enduring impact of not retaining this knowledge from the first course.

TABLE 2

\section{T-TESTS: PERCENT CORRECT ON FINAL EXAM BASED ON REVIEW QUESTIONS CORRECT VS. INCORRECT}

\begin{tabular}{|l|l|c|c|c|c|c|c|}
\hline & $\begin{array}{c}\text { Variance } \\
\text { Assumption }\end{array}$ & $\mathbf{F}$ & $\mathbf{S i g}$. & $\mathbf{t}$ & $\mathbf{f f}$ & $\begin{array}{c}\text { Sig. (2- } \\
\text { tailed) }\end{array}$ & $\begin{array}{c}\text { Mean } \\
\text { Diff- } \\
\text { erence }\end{array}$ \\
\hline R3 Calculate stockholder equity & $\begin{array}{l}\text { Eq. variances } \\
\text { assumed }\end{array}$ & 1.593 & 0.208 & 0.005 & 204 & 0.996 & $0.0 \%$ \\
\hline $\begin{array}{l}\text { R5 Identify asset accounts from } \\
\text { list and total }\end{array}$ & $\begin{array}{l}\text { Eq. variances } \\
\text { assumed }\end{array}$ & $\mathbf{3 . 6 8 5}$ & $\mathbf{0 . 0 5 6}$ & $\mathbf{- 2 . 5 7 0}$ & $\mathbf{2 0 4}$ & $\mathbf{0 . 0 1 1 *}$ & $\mathbf{- 2 . 9 \%}$ \\
\hline $\begin{array}{l}\text { R8 Identify asset accounts from list } \\
\text { and total }\end{array}$ & $\begin{array}{l}\text { Eq. variances } \\
\text { assumed }\end{array}$ & 3.768 & 0.054 & -1.645 & 203 & 0.102 & $-1.8 \%$ \\
\hline $\begin{array}{l}\text { R11 Identify asset accounts from } \\
\text { list and total }\end{array}$ & $\begin{array}{l}\text { Eq. variances } \\
\text { assumed }\end{array}$ & 0.433 & 0.511 & 0.326 & 204 & 0.744 & $0.4 \%$ \\
\hline $\begin{array}{l}\text { R12 Identify asset accounts from } \\
\text { list and total }\end{array}$ & $\begin{array}{l}\text { Eq. variances } \\
\text { assumed }\end{array}$ & 3.745 & 0.054 & 0.948 & 205 & 0.344 & $1.0 \%$ \\
\hline $\begin{array}{l}\text { R13 Select definition of chart of } \\
\text { accounts }\end{array}$ & $\begin{array}{l}\text { Eq. variances } \\
\text { assumed }\end{array}$ & 2.018 & 0.157 & -0.937 & 205 & 0.350 & $-1.0 \%$ \\
\hline $\begin{array}{l}\text { R16 Select a purpose for which a } \\
\text { journal is not useful }\end{array}$ & $\begin{array}{l}\text { Eq. variances } \\
\text { assumed }\end{array}$ & 1.511 & 0.220 & -1.692 & 204 & 0.092 & $-1.7 \%$ \\
\hline $\begin{array}{l}\text { R19 Select the effect a paid } \\
\text { dividend has on accounts }\end{array}$ & $\begin{array}{l}\text { Eq. variances } \\
\text { assumed }\end{array}$ & 0.013 & 0.908 & -1.486 & 203 & 0.139 & $-1.5 \%$ \\
\hline $\begin{array}{l}\text { R33 Select an account increased by } \\
\text { a debit }\end{array}$ & $\begin{array}{l}\text { Eq. variances } \\
\text { assumed }\end{array}$ & 0.275 & 0.601 & -1.063 & 206 & 0.289 & $-1.1 \%$ \\
\hline $\begin{array}{l}\text { R34 Identify credit accounts and } \\
\text { total the amount }\end{array}$ & $\begin{array}{l}\text { Eq. variances } \\
\text { assumed }\end{array}$ & $\mathbf{3 . 0 3 5}$ & $\mathbf{0 . 0 8 3}$ & $\mathbf{- 2 . 8 0 0}$ & $\mathbf{2 0 5}$ & $\mathbf{0 . 0 0 6 * \%}$ & $\mathbf{- 3 . 0 \%}$ \\
\hline
\end{tabular}

*Significant at .05 or better 
TABLE 3

T-TESTS: FINAL COURSE GRADE* BASED ON REVIEW QUESTIONS CORRECT VS. INCORRECT

\begin{tabular}{|l|l|c|c|c|c|c|c|}
\hline & \multicolumn{1}{|c|}{$\begin{array}{c}\text { Variance } \\
\text { Assumption }\end{array}$} & \multicolumn{1}{|c|}{$\mathbf{F}$} & \multicolumn{1}{|c|}{ Sig. } & t & df & $\begin{array}{c}\text { Sig. (2- } \\
\text { tailed) }\end{array}$ & $\begin{array}{c}\text { Mean } \\
\text { Diff- } \\
\text { erence }\end{array}$ \\
\hline R3 Calculate stockholder equity & $\begin{array}{l}\text { Eq. variances } \\
\text { not assumed }\end{array}$ & 4.375 & 0.038 & 0.234 & 202 & 0.815 & 0.09 \\
\hline $\begin{array}{l}\text { R5 Identify asset accounts from } \\
\text { list and total }\end{array}$ & $\begin{array}{l}\text { Eq. variances } \\
\text { assumed }\end{array}$ & 1.696 & 0.194 & -1.537 & 212 & 0.126 & -0.68 \\
\hline $\begin{array}{l}\text { R8 Identify asset accounts from } \\
\text { list and total }\end{array}$ & $\begin{array}{l}\text { Eq. variances } \\
\text { assumed }\end{array}$ & 0.068 & 0.795 & -0.685 & 211 & 0.494 & -0.29 \\
\hline $\begin{array}{l}\text { R11 Identify asset accounts from } \\
\text { list and total }\end{array}$ & $\begin{array}{l}\text { Eq. variances } \\
\text { assumed }\end{array}$ & 1.920 & 0.167 & -0.997 & 212 & 0.320 & -0.42 \\
\hline $\begin{array}{l}\text { R12 Identify asset accounts from } \\
\text { list and total }\end{array}$ & $\begin{array}{l}\text { Eq. variances } \\
\text { assumed }\end{array}$ & 0.073 & 0.788 & 0.223 & 213 & 0.824 & 0.09 \\
\hline $\begin{array}{l}\text { R13 Select definition of chart of } \\
\text { accounts }\end{array}$ & $\begin{array}{l}\text { Eq. variances } \\
\text { assumed }\end{array}$ & 0.260 & 0.611 & 0.713 & 213 & 0.477 & 0.30 \\
\hline $\begin{array}{l}\text { R16 Select a purpose for which a } \\
\text { journal is not useful }\end{array}$ & $\begin{array}{l}\text { Eq. variances } \\
\text { assumed }\end{array}$ & 0.973 & 0.325 & -0.062 & 212 & 0.950 & -0.02 \\
\hline $\begin{array}{l}\text { R19 Select the effect a paid } \\
\text { dividend has on accounts }\end{array}$ & $\begin{array}{l}\text { Eq. variances } \\
\text { assumed }\end{array}$ & 0.553 & 0.458 & -1.337 & 211 & 0.183 & -0.52 \\
\hline $\begin{array}{l}\text { R33 Select an account increased } \\
\text { by a debit }\end{array}$ & $\begin{array}{l}\text { Eq. variances } \\
\text { assumed }\end{array}$ & 0.005 & 0.942 & -1.302 & 214 & 0.194 & -0.54 \\
\hline $\begin{array}{l}\text { R34 Identify credit accounts and } \\
\text { total the amount }\end{array}$ & $\begin{array}{l}\text { Eq. variances } \\
\text { assumed }\end{array}$ & 0.096 & 0.758 & -1.335 & 213 & 0.183 & -0.56 \\
\hline
\end{tabular}

*Letter grade converted to numeric as: $\mathrm{A}+=13, \mathrm{~A}=12, \mathrm{~A}-=11, \mathrm{~B}+=10, \mathrm{~B}=9, \mathrm{~B}-=8, \mathrm{C}+=7, \mathrm{C}=6, \mathrm{C}-=5, \mathrm{D}+=4, \mathrm{D}=3$, $\mathrm{D}-=2, \mathrm{~F}=1, \mathrm{~W}=0$. A mean difference of 1.0 is one third letter grade, e.g., the difference between a $\mathrm{B}+$ and an $\mathrm{A}-$.

Based solely on the Review Quiz, there appears to be minimal impact of these items on overall course performance.

Exam One, given in the sixth week of the course, gives the student another opportunity to demonstrate mastery of selected review items. Six of the 54 questions were the same or similar to those on the review quiz. These are presented in Table 4 and Table 5. 
TABLE 4

T-TESTS: PERCENT CORRECT ON FINAL EXAM BASED ON EXAM ONE QUESTIONS COVERING REVIEW MATERIAL

\begin{tabular}{|l|l|c|c|c|c|c|c|}
\hline & $\begin{array}{c}\text { Variance } \\
\text { Assumption }\end{array}$ & $\mathbf{F}$ & Sig. & $\mathbf{t}$ & $\mathbf{f f}$ & $\begin{array}{c}\text { Sig. (2- } \\
\text { tailed) }\end{array}$ & $\begin{array}{c}\text { Mean } \\
\text { Diff- } \\
\text { erence }\end{array}$ \\
\hline $\begin{array}{l}\text { E1_3 Identify asset accounts } \\
\text { from list and total }\end{array}$ & $\begin{array}{l}\text { Eq. variances } \\
\text { not assumed }\end{array}$ & $\mathbf{1 3 . 5 5 7}$ & $\mathbf{0 . 0 0 0}$ & $\mathbf{- 3 . 9 3 5}$ & $\mathbf{7 4}$ & $\mathbf{0 . 0 0 0 *}$ & $\mathbf{- 5 . 4 \%}$ \\
\hline $\begin{array}{l}\text { E1_52 Select balance sheet } \\
\text { description }\end{array}$ & $\begin{array}{l}\text { Eq. variances } \\
\text { assumed }\end{array}$ & 0.835 & 0.362 & -0.089 & 218 & 0.929 & $-0.2 \%$ \\
\hline $\begin{array}{l}\text { E1_1 Select Income Statement } \\
\text { description }\end{array}$ & $\begin{array}{l}\text { Eq. variances } \\
\text { assumed }\end{array}$ & $\mathbf{1 . 5 2 8}$ & $\mathbf{0 . 2 1 8}$ & $\mathbf{- 2 . 4 3 3}$ & $\mathbf{2 1 8}$ & $\mathbf{0 . 0 1 6} \%$ & $\mathbf{- 3 . 2 \%}$ \\
\hline $\begin{array}{l}\text { E1_53 Calculate stockholder } \\
\text { equity }\end{array}$ & $\begin{array}{l}\text { Eq. variances } \\
\text { assumed }\end{array}$ & 0.347 & 0.556 & -0.142 & 217 & 0.887 & $-0.1 \%$ \\
\hline $\begin{array}{l}\text { E1_8 Select account where debit } \\
\text { balance is normal }\end{array}$ & $\begin{array}{l}\text { Eq. variances } \\
\text { assumed }\end{array}$ & 0.940 & 0.333 & -0.471 & 218 & 0.638 & $-0.5 \%$ \\
\hline $\begin{array}{l}\text { E1_7 Select account where } \\
\text { credit balance is normal }\end{array}$ & $\begin{array}{l}\text { Eq. variances } \\
\text { assumed }\end{array}$ & $\mathbf{2 . 6 5 5}$ & $\mathbf{0 . 1 0 5}$ & $\mathbf{- 2 . 4 7 9}$ & $\mathbf{2 1 8}$ & $\mathbf{0 . 0 1 4} \%$ & $\mathbf{- 2 . 8 \%}$ \\
\hline
\end{tabular}

*Significant at .05 or better.

TABLE 5

T-TESTS: FINAL COURSE GRADE* BASED ON EXAM ONE QUESTIONS COVERING REVIEW MATERIAL

\begin{tabular}{|l|l|c|c|c|c|c|c|}
\hline & $\begin{array}{c}\text { Variance } \\
\text { Assumption }\end{array}$ & $\mathbf{F}$ & Sig. & $\mathbf{t}$ & df & $\begin{array}{c}\text { Sig. (2 } \\
\text {-tailed) }\end{array}$ & $\begin{array}{c}\text { Mean } \\
\text { Diff- } \\
\text { erence }\end{array}$ \\
\hline $\begin{array}{l}\text { E1_3 Identify asset accounts } \\
\text { from list and total }\end{array}$ & $\begin{array}{l}\text { Eq. variances } \\
\text { not assumed }\end{array}$ & $\mathbf{8 . 5 4 7}$ & $\mathbf{0 . 0 0 4}$ & $\mathbf{- 4 . 9 0 8}$ & $\mathbf{7 9}$ & $\mathbf{0 . 0 0 0 * *}$ & $\mathbf{- 2 . 0 3}$ \\
\hline $\begin{array}{l}\text { E1_52 Select balance sheet } \\
\text { description }\end{array}$ & $\begin{array}{l}\text { Eq. variances } \\
\text { assumed }\end{array}$ & 0.000 & 0.983 & -0.305 & 218 & 0.761 & -0.26 \\
\hline $\begin{array}{l}\text { E1_1 Select Income Statement } \\
\text { description }\end{array}$ & $\begin{array}{l}\text { Eq. variances } \\
\text { assumed }\end{array}$ & $\mathbf{0 . 6 3 1}$ & $\mathbf{0 . 4 2 8}$ & $\mathbf{- 3 . 5 3 1}$ & $\mathbf{2 1 8}$ & $\mathbf{0 . 0 0 1 * *}$ & $\mathbf{- 1 . 4 9}$ \\
\hline $\begin{array}{l}\text { E1_53 Calculate stockholder } \\
\text { equity }\end{array}$ & $\begin{array}{l}\text { Eq. variances } \\
\text { assumed }\end{array}$ & 0.011 & 0.915 & -0.934 & 217 & 0.351 & -0.31 \\
\hline $\begin{array}{l}\text { E1_8 Select account where debit } \\
\text { balance is normal }\end{array}$ & $\begin{array}{l}\text { Eq. variances } \\
\text { assumed }\end{array}$ & $\mathbf{1 . 0 8 5}$ & $\mathbf{0 . 2 9 9}$ & $\mathbf{- 2 . 4 4 5}$ & $\mathbf{2 1 8}$ & $\mathbf{0 . 0 1 5 * *}$ & $\mathbf{- 0 . 8 7}$ \\
\hline $\begin{array}{l}\text { E1_7 Select account where } \\
\text { credit balance is normal }\end{array}$ & $\begin{array}{l}\text { Eq. variances } \\
\text { assumed }\end{array}$ & $\mathbf{0 . 0 2 7}$ & $\mathbf{0 . 8 7 1}$ & $\mathbf{- 3 . 9 7 2}$ & $\mathbf{2 1 8}$ & $\mathbf{0 . 0 0 0 * *}$ & $\mathbf{- 1 . 4 3}$ \\
\hline
\end{tabular}

*Letter grade converted to numeric as: $\mathrm{A}+=13, \mathrm{~A}=12, \mathrm{~A}-=11, \mathrm{~B}+=10, \mathrm{~B}=9, \mathrm{~B}-=8, \mathrm{C}+=7, \mathrm{C}=6, \mathrm{C}-=5, \mathrm{D}+=4, \mathrm{D}=3$, $\mathrm{D}-=2, \mathrm{~F}=1, \mathrm{~W}=0$. A mean difference of 1.0 is one third letter grade, e.g., the difference between a $\mathrm{B}+$ and an $\mathrm{A}-$.

**Significant at .05 or better. 
One may expect that gaps in concept mastery that persist well into the course will reflect negatively on course performance. The relationships between review questions on Exam One and the final exam highlight the importance of concepts from the earlier accounting course to success in managerial accounting. The inability to give correct responses to review items on Exam One suggests that the lack of concept retention has continued beyond any initial time when students might begin to think in accounting terms. Specifically, these results suggest that understanding basic accounting concepts such as "What are assets?", "What are debits and credits?", and "What does an income statement show?" are significantly related to the percentage of correct responses on the comprehensive final and to the final course grade. For example, students able to correctly identify and total asset accounts received final course grades that were two thirds of a letter grade higher than those were unable to do so correctly.

\section{Specific Impacts of Deficient Understanding of Basic Material}

We now move on to explore the specific impacts of poor understanding of one central aspect of basic accounting. Based on significance levels and the mean differences, the above analyses suggest that the inability to correctly identify asset accounts (and total them) has a stronger impact compared to other questions which were also significantly related to final outcomes. These results are based on the overall Final Exam score. Here we dive deeper into this specific weakness. Here we consider which concepts within the learning objectives covered in the final exam are related to the inability to correctly identify asset accounts. Table 6 presents analyses using this question from Exam One on final exam questions grouped by learning objectives. We also consider how different incorrect responses students gave on the Exam One question are associated with differences in subsequent success in the course.

These results show clear relationships to overall calculation and definitional questions. These question groupings are too broad to provide much insight although it may be noted that the mean difference on calculation questions $(5.4 \%)$ is three times larger than for the definitional questions $(1.9 \%)$. Even more interesting is that although the ability to correctly identify and total asset accounts on Exam One is unrelated to calculating Material Quantity and Material Price Variances, that ability is clearly related to calculating Labor Quantity and Labor Price Variances. Labor Quantity Variance is significant at $p=0.001$ with students correctly identifying and summing assets having average means on these questions $10.3 \%$ higher than those who were incorrect on Exam One. There were also significant differences on questions related to calculating fixed vs variable cost budgets as well as understanding variable and fixed cost concepts.

TABLE 6

\section{T-TESTS: PERCENT CORRECT ON FINAL EXAM LEARNING CONCEPTS BASED ON EXAM ONE - TOTALING ASSET ACCOUNTS CORRECT VS. INCORRECT}

\begin{tabular}{|c|c|c|c|c|c|c|c|}
\hline & & $\begin{array}{r}\text { Levene } \\
\text { for Equ } \\
\text { Varia }\end{array}$ & $\begin{array}{l}\text { Test } \\
\text { lity of } \\
\text { ices }\end{array}$ & t-tes & for Equ & ality of I & eans \\
\hline & $\begin{array}{l}\text { Variance } \\
\text { Assumption }\end{array}$ & $\mathrm{F}$ & Sig. & $\mathrm{t}$ & df & $\begin{array}{l}\text { Sig. (2- } \\
\text { tailed) }\end{array}$ & $\begin{array}{c}\text { Mean } \\
\text { Diff- } \\
\text { erence }\end{array}$ \\
\hline $\begin{array}{l}\text { Final Course Grade } A=12, B=9, \\
C=6, D=3, F=1, W=0 * *\end{array}$ & $\begin{array}{l}\text { Eq var. not } \\
\text { assumed }\end{array}$ & 8.547 & 0.004 & -4.908 & 78.59 & $0.000 *$ & -2.031 \\
\hline Final Exam \% Correct & $\begin{array}{l}\text { Eq var. not } \\
\text { assumed }\end{array}$ & 13.557 & 0.000 & -3.935 & 73.88 & $0.000 *$ & $-5.4 \%$ \\
\hline All Calculations & $\begin{array}{l}\text { Eq var. not } \\
\text { assumed }\end{array}$ & 4.714 & 0.031 & $-\mathbf{3 . 3 3 5}$ & 77.62 & $0.001 *$ & $-5.7 \%$ \\
\hline All Definitions & $\begin{array}{l}\text { Eq variance } \\
\text { assumed }\end{array}$ & 0.846 & 0.359 & -2.253 & 211 & $0.025 *$ & $-1.9 \%$ \\
\hline
\end{tabular}




\begin{tabular}{|l|c|c|c|c|c|c|c|}
\hline $\begin{array}{l}\text { Calculate Variances Material } \\
\text { Quantity }\end{array}$ & $\begin{array}{c}\text { Eq variance } \\
\text { assumed }\end{array}$ & 0.921 & 0.338 & -0.316 & 211 & 0.752 & $-0.7 \%$ \\
\hline $\begin{array}{l}\text { Calculate Variances Material } \\
\text { Price }\end{array}$ & $\begin{array}{c}\text { Eq variance } \\
\text { assumed }\end{array}$ & 0.315 & 0.575 & -0.714 & 210 & 0.476 & $-2.1 \%$ \\
\hline $\begin{array}{l}\text { Calculate Variances Material } \\
\text { Quantity or Price }\end{array}$ & $\begin{array}{c}\text { Eq variance } \\
\text { assumed }\end{array}$ & 0.059 & 0.808 & -0.685 & 211 & 0.494 & $-1.4 \%$ \\
\hline $\begin{array}{l}\text { Calculate Variances Labor } \\
\text { Quantity }\end{array}$ & $\begin{array}{c}\text { Eq variance } \\
\text { assumed }\end{array}$ & $\mathbf{1 . 1 4 3}$ & $\mathbf{0 . 2 8 6}$ & $\mathbf{- 3 . 2 7 4}$ & $\mathbf{2 1 0}$ & $\mathbf{0 . 0 0 1} \%$ & $\mathbf{- 1 0 . 3 \%}$ \\
\hline $\begin{array}{l}\text { Calculate Variances Labor } \\
\text { Price }\end{array}$ & $\begin{array}{c}\text { Eq var. not } \\
\text { assumed }\end{array}$ & $\mathbf{2 6 . 8 3 9}$ & $\mathbf{0 . 0 0 0}$ & $\mathbf{- 2 . 4 3 6}$ & $\mathbf{7 2 . 0 8}$ & $\mathbf{0 . 0 1 7} \%$ & $\mathbf{- 7 . 8 \%}$ \\
\hline $\begin{array}{l}\text { Calculate Variances Labor } \\
\text { Quantity or Price }\end{array}$ & $\begin{array}{c}\text { Eq var. not } \\
\text { assumed }\end{array}$ & $\mathbf{1 4 . 4 8 3}$ & $\mathbf{0 . 0 0 0}$ & $\mathbf{- 3 . 3 0 7}$ & $\mathbf{7 2 . 1 6}$ & $\mathbf{0 . 0 0 1} \%$ & $\mathbf{- 9 . 3 \%}$ \\
\hline $\begin{array}{l}\text { Calculate Budgets Fixed- } \\
\text { Variable-Total Costs }\end{array}$ & $\begin{array}{c}\text { Eq variance } \\
\text { assumed }\end{array}$ & $\mathbf{1 . 0 3 2}$ & $\mathbf{0 . 3 1 1}$ & $\mathbf{- 2 . 8 1 4}$ & $\mathbf{2 1 0}$ & $\mathbf{0 . 0 0 5}$ & $\mathbf{- 7 . 0 \%}$ \\
\hline $\begin{array}{l}\text { Definition Cost ID Variable- } \\
\text { Fixed-Mixed-Period }\end{array}$ & $\begin{array}{c}\text { Eq variance } \\
\text { assumed }\end{array}$ & $\mathbf{2 . 1 5 0}$ & $\mathbf{0 . 1 4 4}$ & $\mathbf{- 1 . 9 8 5}$ & $\mathbf{2 1 1}$ & $\mathbf{0 . 0 4 8} \%$ & $\mathbf{- 3 . 6 \%}$ \\
\hline $\begin{array}{l}\text { Definition Statements } \\
\text { Distinctions }\end{array}$ & $\begin{array}{c}\text { Eq variance } \\
\text { assumed }\end{array}$ & 2.041 & 0.155 & 0.687 & 210 & 0.493 & $1.0 \%$ \\
\hline $\begin{array}{l}\text { Definition Budgets Distinctions } \\
\text { Eq variance } \\
\text { assumed }\end{array}$ & 1.177 & 0.279 & -0.596 & 210 & 0.552 & $-0.8 \%$ \\
\hline $\begin{array}{l}\text { Definition Management Use of } \\
\text { Accounting }\end{array}$ & $\begin{array}{c}\text { Eq variance } \\
\text { assumed }\end{array}$ & 1.417 & 0.235 & -0.716 & 211 & 0.475 & $-1.4 \%$ \\
\hline Definition Account Interpretation & $\begin{array}{c}\text { Eq var. not } \\
\text { assumed }\end{array}$ & 10.640 & 0.001 & -1.773 & 75.94 & 0.080 & $-3.6 \%$ \\
\hline Definition Account Classification & $\begin{array}{c}\text { Eq var. not } \\
\text { assumed }\end{array}$ & 7.693 & 0.006 & -1.262 & 71.76 & 0.211 & $-1.3 \%$ \\
\hline
\end{tabular}

*Significant at .05 or better.

** Letter grade converted to numeric as: $\mathrm{A}+=13, \mathrm{~A}=12, \mathrm{~A}-=11, \mathrm{~B}+=10, \mathrm{~B}=9, \mathrm{~B}-=8, \mathrm{C}+=7, \mathrm{C}=6, \mathrm{C}-=5, \mathrm{D}+=4, \mathrm{D}=3$, $\mathrm{D}-=2, \mathrm{~F}=1, \mathrm{~W}=0$. A mean difference of 1.0 is one third letter grade, e.g. the difference between a $\mathrm{B}+$ and an $\mathrm{A}-$.

\section{The Distractors}

The students who correctly identified and summed the assets on Exam One did better than those with incorrect responses. There are also differences based on which incorrect distractor was chosen on the question. Although the sum represented by each distractor could be reached by combining a variety of accounts, the simplest combinations are the most likely. Distractor "A" included all asset accounts plus service revenue. Distractor "B" summed Service Revenue and Common Stock accounts. Distractor "C" included all asset accounts except Accounts Receivable. Each distractor implies a different error in response. Table 7 provides a high-level summary of how each distractor compared to the correct Exam One response across the Final Exam summary measures.

Regardless of the distractor chosen by the student there is a significant difference between the letter grade they received in the course and those who chose the correct response. The pattern of success on the summary measures from exam one varies by the distractor chosen. Calculating labor price variance is the only summary measure with significant differences on all three distractors. This is the only summary measure on which distractor "C" (omitting an account) has a significant relationship. Distractor "A" (including an additional account) has significant relationships with more summary measures than distractor "B".

Combining Distractor "A" and "B" students into one group clarified the impact of these errors on the final exam. There is a sharp distinction between their grasping material variances (quantity and price) as well as those with correct responses and labor variances where their percentage correct was significantly lower (10.7\% lower on the combination of labor quantity and price variance questions). Students choosing 
distractors "A" or "B" as well as the combined group had less success on questions related to fixed/variable costs.

TABLE 7

\section{T-TESTS: MEAN DIFFERENCES PERCENT CORRECT ON FINAL EXAM LEARNING CONCEPTS BASED ON EXAM ONE - TOTALING ASSET ACCOUNTS CORRECT VS. DISTRACTORS}

\begin{tabular}{|c|c|c|c|c|c|c|}
\hline & $\begin{array}{l}\text { "A" vs. } \\
\text { Correct* }\end{array}$ & $\begin{array}{l}\text { "B" vs. } \\
\text { Correct* }\end{array}$ & $\begin{array}{l}\text { "C" vs. } \\
\text { Correct* }\end{array}$ & $\begin{array}{c}\text { "A"\&"B" } \\
\text { vs. } \\
\text { Correct* }\end{array}$ & $\begin{array}{c}\mathrm{A} " \& " C " \\
\text { vs. } \\
\text { Correct* }\end{array}$ & $\begin{array}{c}\text { "B"\&"C" } \\
\text { vs. } \\
\text { Correct* }\end{array}$ \\
\hline $\begin{array}{l}\text { Final Course Grade } A=12, B=9, \\
C=6, D=3, F=1, W=0\end{array}$ & $-2.14 * *$ & $-2.85 * *$ & $-1.34 * *$ & $-2.47 * *$ & $-1.7 * *$ & $-1.98 * *$ \\
\hline Final Exam \% Correct & $-7.4 \% * *$ & $-7.6 \% *$ & $-2.3 \%$ & $-7.5 \% * *$ & $-4.6 \% * *$ & $-4.5 \% * *$ \\
\hline All Calculations & $-8 \% * *$ & $-6.1 \% * *$ & $-3.6 \%$ & $-7.2 \% * *$ & $-5.6 \% * *$ & $-4.6 \% * *$ \\
\hline All Definitions & $-5 \% * *$ & $-0.7 \%$ & $-0.1 \%$ & $-3.1 \% * *$ & $-2.3 \% * *$ & $-0.3 \%$ \\
\hline $\begin{array}{l}\text { Calculate Variances Material } \\
\text { Quantity }\end{array}$ & $-2.6 \%$ & $2.8 \%$ & $-1.5 \%$ & $-0.3 \%$ & $-2.0 \%$ & $0.2 \%$ \\
\hline $\begin{array}{l}\text { Calculate Variances Material } \\
\text { Price }\end{array}$ & $-0.3 \%$ & $0.3 \%$ & $-5.1 \%$ & $0.0 \%$ & $-2.9 \%$ & $-3.0 \%$ \\
\hline $\begin{array}{l}\text { Calculate Variances Material } \\
\text { Quantity or Price }\end{array}$ & $-1.6 \%$ & $1.7 \%$ & $-3.1 \%$ & $-0.2 \%$ & $-2.4 \%$ & $-1.2 \%$ \\
\hline $\begin{array}{l}\text { Calculate Variances Labor } \\
\text { Quantity }\end{array}$ & $-10.8 \% * *$ & $-12.5 \% * *$ & $-8.5 \% * *$ & $-11.5 \% * *$ & $-9.5 \% * *$ & $-10.1 \% * *$ \\
\hline Calculate Variances Labor Price & $-6.9 \%$ & $-11.1 \%$ & $-6.5 \%$ & $-8.8 \% * *$ & $-6.7 \%$ & $-8.3 \% * *$ \\
\hline $\begin{array}{l}\text { Calculate Variances Labor } \\
\text { Quantity or Price }\end{array}$ & $-9.6 \%$ & $-12.2 \%$ & $-7.3 \%$ & $-10.7 \% * *$ & $-8.4 \% * *$ & $-9.2 \% * *$ \\
\hline $\begin{array}{l}\text { Calculate Budgets Fixed- } \\
\text { Variable-Total Costs }\end{array}$ & $-15.1 \% * *$ & $-8.8 \% * *$ & $0.8 \%$ & $-12.3 \% * *$ & $-6.3 \% * *$ & $-2.9 \%$ \\
\hline $\begin{array}{l}\text { Definition Cost ID Variable- } \\
\text { Fixed-Mixed-Period }\end{array}$ & $-10.7 \% * *$ & $-1.5 \%$ & $0.8 \%$ & $-6.7 \% * *$ & $-4.3 \% * *$ & $-0.1 \%$ \\
\hline $\begin{array}{l}\text { Definition Statements } \\
\text { Distinctions }\end{array}$ & $4.3 \% * * *$ & $0.7 \%$ & $-1.4 \%$ & $2.7 \% * * *$ & $1.1 \%$ & $-0.6 \%$ \\
\hline Definition Budgets Distinctions & $-5.6 \%$ & $2.3 \% * * *$ & $1.1 \%$ & $-2.2 \%$ & $-1.9 \%$ & $1.6 \%$ \\
\hline $\begin{array}{l}\text { Definition Management Use of } \\
\text { Accounting }\end{array}$ & $-4.7 \%$ & $0.4 \%$ & $0.2 \%$ & $-2.4 \%$ & $-2.0 \%$ & $0.3 \%$ \\
\hline $\begin{array}{l}\text { Definition Account } \\
\text { Interpretation }\end{array}$ & $-4.6 \%$ & $-4.3 \%$ & $-2.3 \%$ & $-4.4 \%$ & $-3.3 \%$ & $-3.1 \%$ \\
\hline $\begin{array}{l}\text { Definition Account } \\
\text { Classification }\end{array}$ & $-3.9 \%$ & $-1.0 \%$ & $0.5 \%$ & $-2.6 \%$ & $-1.5 \%$ & $-0.1 \%$ \\
\hline
\end{tabular}

*Distractor "A" is Assets plus Service Revenue, "B" is Service Revenue plus Common Stock, and "C" is Assets omitting Accounts Receivable.

**Students choosing this distractor had a significantly $(\mathrm{p}<.05)$ lower percent correct compared to those choosing the correct response. The difference in mean percent correct is shown in the table.

***Note the reverse direction of this relationship. Students choosing this distractor had a significantly $(\mathrm{p}<.05)$ higher percent correct compared to those choosing the correct response. 


\section{DISCUSSION}

A student's ability to correctly identify and total asset accounts is a knowledge and skill which should carry over from an entry accounting course to managerial accounting. We have shown evidence that students who are unable to demonstrate this skill by the first exam of the managerial course are less successful on their final exam and in their final course grade. Some learning objectives in the course appear to be differentially affected with labor variances and variable/fixed cost concepts showing greater differences. Additional research will be needed to identify the nature of these linkages to some groups of concepts yet not to others.

How might we use these findings to improve student success? Focusing on the introductory course seems unlikely to yield a strong result because of the length of time between courses and the different institutions where the introductory course may have been taken. We believe stronger student performance may come from holding required review sessions prior to Exam One. Alternatively, holding follow-up sessions with selected students based on Exam One results might help.

\section{REFERENCES}

AECC (Accounting Education Change Commission). (1992). The First Course in Accounting: Position Statement No. Two. Issues in Accounting Education, 7(2), 249-251.

AAA (American Accounting Association). (1986). Committee on the Future Structure, Content and Scope of Accounting Education (The Bedford Committee). Special Report: Future Accounting Education: Preparing for the Expanding Profession. Issues in Accounting Education, 1(1), 168193.

Arthur Andersen \& Co., Arthur Young, Coopers \& Lybrand, Deloitte Haskins and Sells, Ernst \& Whinney, Peat Marwick Main \& Co., Price Waterhouse, Touche Ross. (1989). Perspectives on Education: Capabilities for Success in the Accounting Profession. New York: Authors.

Baldwin, B.A., \& Ingram, R.W. (1991). Rethinking the Objectives and Content of Elementary Accounting. Journal of Accounting Education, 9(1), 1-14.

Byrne, M., \& Flood, B. (2008). Examining the Relationships Among Background Variables and Academic Performance of First Year Accounting Students at an Irish University. Journal of Accounting Education, 26(4), 202-212.

Cherry, A.A., \& Mintz, S.M. (1996). The Objectives and Design of the First Course in Accounting from the Perspective of Non-Accounting Faculty. Accounting Education - A Journal of Theory, Practice \& Research, 1(2), 99-111.

Cherry, A.A., \& Reckers, P.M.J. (1983). The Introductory Financial Accounting Course: Its Role in the Curriculum for Accounting Majors. Journal of Accounting Education, 1(1), 71-82.

Danko, K., Duke, J.C., \& Franz, D.P. (1992). Predicting Student Performance in Accounting Classes. Journal of Education for Business, 67(5), 270-274.

Doran, B.M., Bouillon, M.L., \& Smith, C.G. (1991). Determinants of student performance in accounting principles I and II. Issues in Accounting Education, 6(1), 74-84.

Geiger, M.A., \& Ogilby, S.M. (2000). The First Course in Accounting: Students' Perceptions and Their Effect on the Decision to Major in Accounting. Journal of Accounting Education, 18, 63-78.

Jordan, E.E., \& Samuels, J.A. (2020). Research Initiatives in Accounting Education: Improving Learning Effectiveness. Issues in Accounting Education, 35(4), 9-24.

Kaenzig, R., \& Keller, R. (2011). A Comprehensive Effort to Recruit and Retain Accounting Students. Journal of Accounting Education, 29, 63-78.

Pasewark, W.R. (2020). Research Initiatives in Accounting Education: A Proposed Agenda for Accounting Education Research. Issues in Accounting Education, 35(4), 3-7.

Pincus, K.V. (1997). Is Teaching Debits and Credits Essential in Elementary Accounting? Issues in Accounting Education, 12(2), 575-579. 
Pincus, K.V., Stout, D.E., Sorenson, J.E., Stocks, K.D., \& Lawson, R.A. (2017). Forces for Change in Higher Education and Implications for the Accounting Academy. Journal of Accounting Education, 40, 1-18.

Papageorgiou, E., \& Carpenter, R. (2019). Prior Accounting Knowledge of First-Year Students at Two South African Universities: Contributing Factor to Academic Performance or Not? South African Journal of Higher Education, 33(6), 249-264.

Papageorgiou, K., \& Halabi, A. K. (2014). Factors contributing toward student performance in a distance education accounting degree. Meditari Accountancy Research, 22(2), 211-223.

Tan, L.M., \& Laswad, F. (2008). Impact of Prior Content and Meta-Cognitive Knowledge on Students' Performance in an Introductory Accounting Course. Pacific Accounting Review, pp. 63-74.

Vangermeersch, R.G. (1997). Dropping Debits and Credits in Elementary Accounting: A Huge Disservice to Students. Issues in Accounting Education, 12(2), 581-583.

Warren, D.L., \& Young, M.N. (2012). Integrated Accounting Principles: A Best Practices Course for Introductory Accounting. Issues in Accounting Education, 27(1), 247-266. 